\title{
Uso y aplicación de herramientas 2.0 en los servicios, producción, organización y difusión de la información en la biblioteca universitaria
}

\author{
Julio Alonso Arévalo \\ José Antonio Cordón García \\ Raquel Gómez Díaz * \\ Belén García-Delgado Giménez **
}

Artículo recibido:

28 de enero de 2013.

Artículo aceptado:

27 de febrero de 2014.

\section{Resumen}

El presente estudio tiene como objetivo presentar un análisis sobre el uso de herramientas 2.0 en bibliotecas universitarias, con el fin de entender los patrones de uso como una forma de proporcionar información a los usuarios y mejorar la visibilidad de la entidad a través de su marca digital. El análisis, basado en la experiencia profesional, muestra cómo la integración de diferentes herramientas 2.0 permite potenciar el servicio de información que ofrece la biblioteca universitaria al tiempo que favorece la creación de comunidades de aprendizaje y fomenta los mecanismos de comunicación entre los usuarios. La integración de los

* Los tres autores pertenecen a la Universidad de Salamanca, España. alar@usal.es; jcordon@usal.es; rgomez@usal.es

** Universidad Europea de Madrid, España. belen.garcia-delgado@uem.es

INVESTIGACIÓN BIBLIOTECOLÓGICA, Vol. 28, Núm.64, septiembre/diciembre, 2014 , México, ISSN: 0187-358X. pp. 51-74 
distintos tipos de herramientas de la web social permite articular un sistema de información, donde gracias a sistemas como los canales RSS (Really Simple Syndication) se facilita la recopilación de información, la cual es organizada y normalizada con los gestores de referencias sociales y difundida entre los usuarios a través de los blogs, redes sociales y listas de distribución, lo que permite darle una mayor visibilidad a la institución, pero sobre todo ofrecer mejores servicios a los usuarios. El valor del artículo radica en que la experiencia de un centro concreto permite su réplica y/o adaptación a otros centros en los que se podrán implantar servicios similares, puesto que los servicios proporcionados se basan en herramientas de la web 2.0, freeware o de código abierto.

Palabras clave: España; Biblioteca 2.0; Bibliotecas académicas; Cambio sociotécnico; Freeware; Código abierto; Identidad digital.

\section{Abstract}

Use and application of tools 2.0 in information organization and dissemination services in the university library

Julio Alonso-Arévalo, José-Antonio Cordón-García, Raquel Gómez-Diaz and Belén García-Delgado Giménez

The object of this study is to present an analysis on the use of 2.0 tools in university libraries, with the goal of grasping usage patterns of the information provided to users, while enhancing the visibility of the entity's digital brand. Based on professional experience, the analysis shows how the integration of diverse 2.0 tools can improve the information services offered by the university library, while improving communication mechanisms among users. This encourages the creation of learning communities. The integration of diverse social networking tools allows for the articulation of an information system, in which, thanks to RSS feeds channels, information is organized and standardized by social reference managers and subsequently disseminated among users through blogs, social networks, distribution lists; thereby enhancing the institution's visibility and, above all, providing bet- 
portrait of an information center, using 2.0 web tools, freeware and open code application; whose experience can be replicated and/or adapted in other centers, where similar services are offered

Keywords: Spain; Library 2.0; Academic Libraries; Sociotechnical Change; Freeware; Open Code; Digital Identity.

$$
* * *
$$

The principles of Library 2.0 seek to put users in touch with information and entertainment wherever they may be, breaking down the barriers of space, time and outdated policy.

It is a user-centered paradigm focusing on knowledge, experience, collaboration, the creation of new content and encouraging the beart.

Michael Stephens http://www.tametheweb.com/

\section{UNA BREVE REVISIÓN BIBLIOGRÁFICA DE LA BIBLIOTECA EN EL CONTEXTO 2.0}

T a aplicación de las tecnologías 2.0 en los servicios bibliotecarios ha teUnido una repercusión muy importante en los propios servicios, como se muestra en la abundante literatura profesional existente, donde se abordan prácticamente todos los aspectos relacionados con aquéllos y su capacidad para difundir y el impacto que están teniendo en las bibliotecas las herramientas (Aharony, 2009; Cordón-García et al., 2012b; Chapman \& Russell, 2009; Noa, 2012; Rasmussen, 2009; Rozaklis \& MacDonald, 2011) y aplicaciones de la web 2.0 en los entornos bibliotecarios (Alonso Arévalo et al., 2010; Cordón García et al., 2012a; Cordón García et al., 2012c; Harris, 2008; Kroski, 2008); incluso algunos autores se han llegado a plantear la reformulación del propio concepto de biblioteca (Cooke, 2011; Chad \& Miller, 2005; Daniels \& Roth, 2012; Holmberg et al., 2009; Miller, 2005; Wilson, 2012) y del profesional, abordando la necesidad de capacitar a éste en las nuevas competencias para el desarrollo y manejo de estas tecnologías (Castagné, 2011; Feng, 2011; González-Fernández Villavicencio, 2007; Nguyen et al., 2012; Quinney et al., 2010; Saha et al., 2008 y Stephens, 2006).

Igualmente es profusa la bibliografía que ha estudiado la influencia de la web 2.0 en el ámbito académico bajo la perspectiva de la Ciencia 2.0 y sus ca- 
pacidades para difundir la información (Armstrong, 2011; Jacsó, 2011; Priem \& Hemminger, 2010; REBIUN, 2011; Spring, 2011).

Todos estos trabajos subrayan el hecho de que la práctica bibliotecaria está cambiando, y que la integración de las distintas herramientas en las actividades de la biblioteca favorece un mejor aprovechamiento de los recursos.

\section{El CONCEPTO 2.0}

Durante los últimos años las innovaciones tecnológicas han transformado la manera en que se procesa, almacena, accede, comparte y analiza la información. Los papeles tradicionales asignados al productor, al procesador y al usuario de la información están experimentando también importantes transformaciones. El universo de la información está cambiando de manera vertiginosa y las bibliotecas deben responder de manera eficaz a estos cambios para adaptarse a las necesidades de la sociedad a la que sirven y ofrecer los recursos y servicios que los usuarios necesitan y desean.

En el momento actual nos encontramos con nuevos entornos apoyados en la tecnología. Los servicios son cada vez más participativos y en ellos se busca la colaboración de los usuarios y abrir nuevas vías para ganar autoridad y reputación digital. Por otro lado, el centro de atención se está desplazando de la colección actual, la física, a la colección virtual, o dicho de otra manera, se potencia más la información que el soporte en el que está contenida la misma. En esta transición el usuario va adquiriendo cada vez más importancia, y en lugar de darle un servicio se busca integrarlo en el propio servicio. De este modo la disponibilidad, flexibilidad y movilidad de la información a través de conexiones y redes va ganando cada vez más relevancia. En este entorno el bibliotecario y el usuario se integran y colaboran en el desarrollo y difusión de la "colección", de la información y del conocimiento. Y si bien esto se puede dar en cualquier tipo de biblioteca, en el caso de las de enseñanza superior adquiere una mayor importancia, ya que la implicación del usuario en el desarrollo de los sistemas de aprendizaje convierte a la biblioteca en uno de los ejes clave del sistema. Pero para conseguirlo es necesario reorganizar las tareas de gestión, organización y difusión, así como los propios servicios de información, que le permitirán a la biblioteca implementar servicios más eficientes y competitivos.

Las TIC han fortalecido y favorecido la capacidad de proporcionar servicios bibliotecarios en términos de calidad y capacidad para llegar a más personas. La Web es un medio cada vez más participativo, donde la gente ya no sólo quiere consultar, sino que además quiere opinar, comunicarse y crear. Se trata 
por tanto de una suerte de conversación global, donde el usuario ya no quiere ser sólo un consumidor pasivo; cualquiera tiene algo que decir y también desea tener a alguien que lo escuche. Por otro lado, las entidades necesitan ampliar la base social de usuarios y mejorar su reputación e identidad digital.

El concepto 2.0 adquiere una dimensión que recuerda más a una aplicación informática de segunda generación, gracias al uso de herramientas participativas que fomentan la implicación activa del usuario, siendo este último el centro de atención fundamental, tal y como subrayan Michael Casey y Laura C. Savastinuk (2006: s. pág.): "The heart of Library 2.0 is user-centered change. It is a model for library service that encourages constant and purposeful change, inviting user participation in the creation of both the physical and the virtual services they want, supported by consistently evaluating services". ${ }^{1}$ Esta misma idea se recoge entre la declaración de intenciones del popular A Librarian's 2.0 Manifesto de Laura Cohen (Tripathi \& Kumar, 2010).

Por lo tanto podemos decir que la filosofía 2.0 ofrece a las bibliotecas no sólo la gran oportunidad de acercarse aún más a sus usuarios, conocer qué les interesa y qué necesitan, y ofrecérselo de la forma que mejor se adapte a sus intereses, sino que también permite integrar a los usuarios como un elemento más del sistema de información, porque ahora el propio usuario también genera información.

Los aspectos clave de la biblioteca 2.0 pueden definirse en los siguientes puntos (Vállez \& Marcos, 2009):

1. Se centra en el usuario.

2. Busca patrones que le den cabida a la mayoría de los usuarios.

3. Sus contenidos provienen de diferentes fuentes y deben facilitar su integración.

4. Intenta hacer uso de la inteligencia colectiva

5. Suele requerir la integración de diferentes tipos de software.

La biblioteca 2.0 es por tanto un espacio virtual en el que interactuarán no sólo las fuentes de información, sino también las herramientas tecnológicas y los sistemas de metadatos, de recuperación y de soporte humano en un ambiente adaptado. El uso de las herramientas 2.0 está cada vez más extendido y según la mayoría de los estudios que han analizado y evaluado el uso de este tipo de herramientas en el entorno bibliotecario, son los blogs y las redes

1 "La esencia de Library 2.0 es el cambio centrado en el usuario. Se trata de un modelo de servicios bibliotecarios que fomenta el cambio constante, pero con sentido de dirección y propósito, que invita al usuario a participar en la creación de los servicios físicos y virtuales que desea, apoyado en todo momento por servicios sometidos a evaluación permanente". (N. del E.) 
sociales los que se utilizan con mayor frecuencia, y en menor medida otro tipo de herramientas que se pueden denominar tecnología de segundo nivel, como los marcadores sociales, los podcast y las wikis (Kim \& Abbas, 2010).

Una de las características de las herramientas del entorno 2.0 es su facilidad de uso. Normalmente las destrezas informáticas que requieren son las de cualquier usuario medio. Para la biblioteca el objetivo reside, como apuntan Vállez y Marcos (2009), en que la integración de este software sirva para generar un sistema de información válido y comprensible para cualquier tipo de usuario. Ésa es precisamente la clave para poder articular estos recursos y herramientas de manera integral y generar un servicio de información que, además, contribuya a mejorar la visibilidad y optimizar la marca digital de la biblioteca en su contexto social, así como a fomentar la creación de comunidades y mecanismos de comunicación y aprendizaje entre los usuarios.

\section{Herramientas de LA BIBLIOTECA UNIVERSITARIA 2.0}

En este apartado se analizarán algunas de las herramientas que se utilizan habitualmente desde las tres perspectivas establecidas anteriormente. En el primer grupo se encuentran aquellas que facilitan a la biblioteca la recopilación de la información, como los canales RSS o las revistas electrónicas que integran funcionalidades de la web social; en el segundo están aquellas herramientas que posibilitan la organización de los recursos, como son los gestores de referencias sociales y las herramientas de gestión bibliográfica como Zotero, CiteUlike o Mendeley, y en el tercero, aquellas otras que se utilizan para la difusión de la información, como son los blogs, las listas de distribución y las redes sociales (Tajer, 2009).

A pesar de esta clasificación, es necesario apuntar que hay algunas herramientas que se utilizan con un carácter polivalente, es decir, que tienen una doble funcionalidad, y un sentido bidireccional. Esto significa que, en un momento inicial, pueden servir para recopilar información de utilidad para la comunidad, y posteriormente pueden constituirse en canal de difusión para los distintos recursos, como ocurre con los canales RSS; asimismo existen herramientas que integran las tres funciones, como sucede con los gestores de referencias bibliográficas, que permiten la recolección de información, su organización y su difusión (Figura 1). Incluso hay herramientas similares que se pueden utilizar para abrir diferentes canales de difusión en función de las cualidades de los usuarios que las utilizan, como son las redes sociales, que pueden ser genéricas como Twitter o Facebook o especializadas como Research ID, Academia.edu, etcétera. 


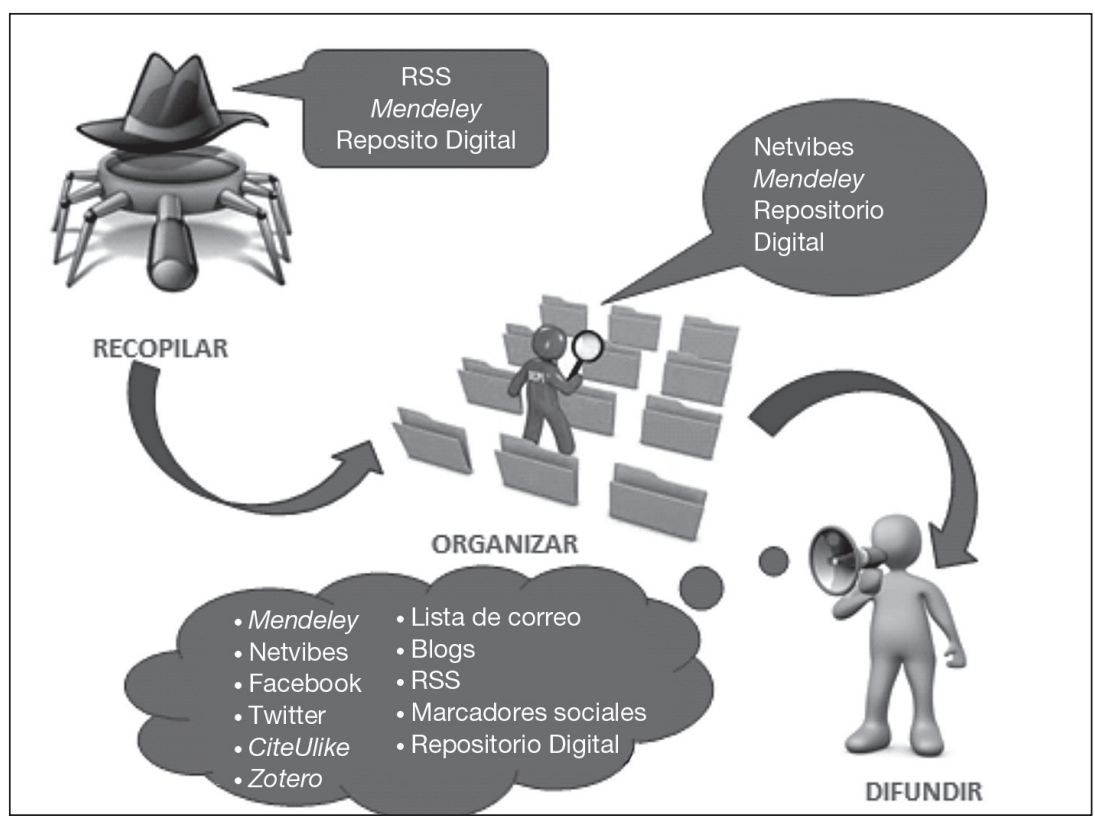

Figura 1. Herramientas de la Biblioteca Universitaria 2.0

\section{Herramientas para la recopilación de información}

\section{RsS (Really Simple Sindication)}

Una de las herramientas más potentes para la recopilación y difusión de la información son los canales RSS (Really Simple Sindication), que permiten disponer de las actualizaciones de una web sin necesidad de navegar de página en página o repetir la misma búsqueda de manera periódica. Gracias a estos canales se multiplica la capacidad de gestionar la información al permitir la automatización de su búsqueda. Estos canales permiten dirigir el tráfico de información hacia una especie de tablón de anuncios electrónico, que proporciona un resumen sobre una noticia y que a través de un enlace se puede ampliar. En este proceso un productor o un distribuidor de contenidos le facilita información en formato digital a un suscriptor, generalmente con el ánimo de que dichos contenidos sean integrados en sus navegadores o en sus propios sitios web. Estas noticias se pueden leer directamente en la página web o bien mediante un agregador de contenidos como Feedreader (http:// feedreader.com/) o Google Reader (http://www.google.com/reader). Se trata de una herramienta que sirve tanto para la fase de recopilación de la información como para la fase de difusión. 


\section{Gestores de referencias sociales}

Fueron diseñados inicialmente como herramientas orientadas al trabajo de los investigadores y posibilitan que éstos puedan disponer de un mecanismo fácil de utilizar para compilar, organizar y citar referencias bibliográficas en los trabajos de investigación. Sin embargo, los gestores de referencias son una excelente herramienta orientada también hacia fines profesionales para promover un servicio de información adaptado a las necesidades de la biblioteca y de los usuarios más exigentes. Las herramientas de primera generación fueron los gestores que comercializaba Thompson Inc. como ProCite, EndNote y Reference Manager. A éstos les han seguido herramientas de segunda generación como RefWorks o Zotero, que incorporan funcionalidades web y, finalmente, una tercera generación basada en las funciones 2.0 que permiten compartir recursos y generar auténticas redes sociales en torno a un asunto, como ocurre, por ejemplo, con Mendeley y CiteUlike. En gran medida el caso que desarrollamos en este estudio se basa en las funciones de estas herramientas que desde la biblioteca se combinan con otros recursos de las web 2.0.

\section{Herramientas para organizar y generar nueva información}

Como se afirmaba al principio del documento, la nueva biblioteca tiene que ser proactiva en el sentido de evolucionar y adaptarse a los nuevos usuarios y a las necesidades manifestadas por ellos. El bibliotecario del siglo XXI deber tener una actitud abierta a su entorno e ir integrando los nuevos sistemas de información en los que interactúan los usuarios. Han sido varios los autores que han planteado nuevas competencias en el desarrollo profesional del bibliotecario como editor y generador de nuevo conocimiento (Baiget, 2010).

En esta misma sección se podrían haber incluido los gestores de referencias bibliográficas, ya que no sólo facilitan recopilar información, sino que además permitirían organizarla e incluso difundirla. En este sentido herramientas como Mendeley, Zotero y CiteUlike permiten crear comunidades de referencias compartidas entre investigadores o grupos de usuarios. Igual podríamos decir de las aplicaciones de escritorio como Netvibes (Méndez González, 2012).

\section{Mashups y aplicaciones de escritorio}

Existen diferentes aplicaciones de escritorio, quizás la más conocida sea Google+, aunque probablemente la que mayor reconocimiento y aplicación ha tenido en bibliotecas es Netvibes, desarrollada por Tariq Krim en 2005. 
Ha sido una de las herramientas pioneras de la Web 2.0 en ofrecer escritorios personalizados al estilo de Google+. Se diferencia de otras aplicaciones de escritorio personalizado por tener una parte pública y otra privada. Netvibes permite reunir widgets favoritos, sitios web, blogs, cuentas de correo electrónico, redes sociales, motores de búsqueda, servicios de mensajería instantánea, canales RSS, fotos, videos y podcasts. Lo anterior permite que sea utilizada como una guía de recursos de la biblioteca, como LibGuides.

\section{Herramientas para difundir información y canales para generar la comunicación}

\section{Listas de correo}

Las listas de correo fueron las primeras formas de comunicación social en Internet. La Web ha ido integrando en su estructura la mayoría de las herramientas de la primera Internet. Sin embargo, a pesar de su mundo deslumbrante de imágenes y colorido, el correo electrónico ha sido la única herramienta que se ha mantenido por utilidad y facilidad de uso tanto en la comunicación individual como en la colectiva a través de las listas de discusión y distribución. Una lista de distribución consiste en agrupar de un conjunto de direcciones de correo electrónico de personas interesadas en un tema específico y común, de modo que se puedan enviar mensajes simultáneamente a todas ellas utilizando una única dirección. De este modo, cuando un usuario envía un correo a una lista este mensaje se envía automáticamente a todos los miembros que están suscritos a la misma. Las listas de correo siguen teniendo una alta validez por ser herramientas fundamentales debido a su dinamismo para la comunicación, tanto externa como interna, entre los miembros de una red. Algunos de estos servicios están basados en sistemas de código abierto como Mailman (http://www.gnu.org/software/mailman/ index.html). Se trata de una herramienta que posibilita una alta capacidad de difusión con un bajo coste y que permite dirigir la información a usuarios con un nivel de interés concreto (Alonso Arévalo \& Vázquez Vázquez, 2013).

\section{Blogs}

Los blogs son un medio útil para la divulgación de la información, ya que proporcionan noticias interesantes o curiosas sobre un asunto, aspecto que puede ser más "atractivo" para personas que no quieren o no desean consultar otro tipo de fuentes. Los blogs tienen una serie de características que los definen: 
- Formato de publicación en línea.

- Configuración cronológica inversa.

- Estructura de diario.

- Contenidos: enlaces, noticias y opiniones.

- Autoría mayoritariamente individual.

- Un estilo informal y subjetivo.

- Posibilidad de incluir comentarios y sindicar (o asociar) contenidos.

Su razón de ser obedece a motivaciones muy diversas que van desde el uso para una más ágil comunicación profesional en entidades hasta la voluntad de compartir información, opinar, crear diarios personales, establecer relaciones públicas, difundir notas, pensamientos, ideas o simplemente porque sí. Los blogs gozan de gran popularidad, de hecho la blogosfera global se duplica cada 5 meses, aunque hay que tener en cuenta que la tasa de abandono es también muy alta. En conclusión se trata de una herramienta muy eficiente ya que es de uso fácil y sencillo, además de resultar muy dinámica al facilitar la comunicación con los usuarios.

\section{Redes sociales}

Una red social es un sitio web que permite a los usuarios compartir contenido, interactuar y crear comunidades sobre intereses similares. Las redes sociales probablemente han sido uno de los fenómenos que han tenido un mayor impacto en los últimos años, tanto en los aspectos relativos a las relaciones personales como profesionales. Estas herramientas han multiplicado las capacidades de comunicación personal y las posibilidades de recepción de información entre las personas. Podríamos hablar de dos grandes tipos: generales, como Facebook o Twitter, y especializadas como Academia.edu (http://www. academia.edu/), ResearchGate (http://www.researchgate.net/) o Librarything (http://www.librarything.es). Las redes sociales son un magnífico escaparate para ofrecer los servicios bibliotecarios a los usuarios, y para que desde la biblioteca se organicen comunidades en torno a sus temas de interés, que irían desde la lectura social con recomendaciones de libros entre usuarios hasta la creación de grupos vinculados con los temas de interés del centro.

LA INTEGRACIÓN DE LAS HERRAMIENTAS 2.0 EN UN SISTEMA DE INFORMACIÓN

En esta parte se pretende mostrar un caso práctico de integración de distintas herramientas de la web 2.0 que permiten generar un servicio completo de 
información y apreciar cómo su uso apoya y facilita las tareas de recolección de información, organización, elaboración y difusión de contenidos. El caso que nos ocupa se basa en los servicios generados por la Biblioteca de la Facultad de Traducción y Documentación de la Universidad de Salamanca (España). Se trata por tanto de una biblioteca de centro que a su vez es un punto de servicio más de los que ofrece a su comunidad la Universidad de Salamanca. Atiende aproximadamente a un millar de usuarios presenciales, cifra que no representa mas que una mínima parte de sus usuarios reales, pues gracias a la capacidad participativa de estas herramientas se ha podido integrar a un gran número de usuarios que, sin acudir al centro, se benefician de los servicios que la biblioteca ofrece. La capacidad de impacto de la biblioteca sobre la comunidad profesional en el entorno virtual es importante porque son miles de usuarios los que utilizan sus servicios y consultan sus recursos. Por dar una idea de estas cifras el blog de la biblioteca, Universo Abierto (http://www.universoabierto.com/), recibió 270000 visitas en el año 2011, que permanecen en la página consultada al menos durante dos minutos, tomando los datos proporcionados por una herramienta externa como Google Analytics. La lista de distribución llamada InfoDoc tiene más de 7 000 suscriptores y lleva en funcionamiento 15 años. Los grupos en Facebook creados por la biblioteca integran a más de 6000 miembros.

La influencia generada por los medios sociales ha sido calificada como Investigación 2.0, Social Reference o Altmetrics, entre otros términos. Varios autores han investigado la influencia en Twitter, en blogs y en gestores de referencias (Jiepu Jiang \& Ni, 2011). Sin lugar a dudas la mayor parte de los investigadores ha trasladado sus actividades de investigación a la Web. Y con el éxito de los medios sociales esta situación se ha hecho más evidente, ya que estas herramientas tienen un gran potencial para ejercer y desarrollar mayor influencia académica que los entornos tradicionales de publicación. A pesar de que todavía las publicaciones impresas siguen teniendo una fuerte influencia en la comunidad académica, los medios sociales como blogs, repositorios, redes sociales y gestores de referencias en línea están empezando a ser tenidos en cuenta en la valoración de las publicaciones porque ofrecen una imagen más completa acerca del impacto de éstas. Sitios como Google Scholar incluyen muchos tipos documentales como preprints, presentaciones, artículos o tesis que no aparecen en los sistemas tradicionales como Web of Science o Scopus, y que de alguna manera reflejan una más amplia tipología del impacto de los autores (Aguillo, 2009).

Bibliotecas digitales, Repositorios de Objetos de Aprendizaje (ROA), Recursos Educativos Abiertos (REA), revistas y bases de datos de artículos permiten descargar un documento y disponer de estadísticas de uso; todas ellas 
favorecen la popularidad de los artículos y por tanto su potencial de lectura, lo que sin ninguna duda constituye un buen indicador de su valor o influencia científica. Algunos estudios también han establecido la correlación entre el número de visitas y descargas a un documento y el número de citas recibidas, lo que sugiere de alguna manera que las estadísticas de uso tienen una relación directa con su impacto (Brody, 2006). Es necesario considerar que esos sistemas también entrañan algunos problemas prácticos respecto al uso de estas estadísticas para evaluación de la investigación, como la falta de uniformidad y normalización, o que los propios editores las manipulen para obtener una mayor influencia. Las mayores críticas se centran en argumentar que se trata de una simple medida de uso y no de hecho de la influencia científica (Neylon, 2011). Una serie de investigadores han trabajado en la identificación de nuevos métodos cuantitativos de evaluación de la investigación para la Web con el objetivo de complementar el análisis de citas tradicional (Burgelman et al., 2010; Priem et al., 2011; Thelwall, 2008). Como se indica en el manifiesto Almetrics (http://altmetrics.org/manifesto/), es preciso encontrar nuevos métodos de medición más acordes con la realidad documental y la práctica de investigación actual.

\section{Génesis y mantenimiento del servicio}

La génesis y construcción del servicio parte de la adopción de algunas herramientas básicas de gran potencial, como es el caso de los primeros gestores de referencias, que sirvieron para establecer un sistema regular de recolección de datos a través de diferentes fuentes de información. Estas fuentes ayudaron a conformar una base de datos propia que actualmente cuenta con más de 50000 referencias de texto completo. Posteriormente se integraron y articularon otras herramientas al sistema de información. Pero la base de datos necesita mecanismos de difusión, por ello se consideró la creación de una lista de distribución, un mecanismo de gran validez y vigencia actual que puede ser considerado de alguna manera como una de las primeras formas de interacción social de la Web. Este entramado inicial de servicios de información y lista de distribución tuvo una muy importante aceptación -9700 suscriptores-, ya que era un servicio pionero que sirvió de base y marca para difundir los otros servicios que se implementaron posteriormente como la página web, blogs, el inicio del proyecto DoIS -que posteriormente evolucionó en el repositorio E-LIS-, gestores de referencias sociales, redes sociales y grupos en Facebook.

Respecto al mantenimiento e inversión en el proyecto, es necesario mencionar que han sido mínimos a lo largo de estos más de 20 años de servicio, 
puesto que se han utilizado fundamentalmente recursos gratuitos disponibles en la Web, bien basados en software libre, freeware o mantenidos por otras instituciones (listas de correo). De este modo el costo para la entidad en la que se prestan los servicios, en este caso la Universidad de Salamanca, ha sido mínimo. Actualmente existen dos gastos extras: el mantenimiento de una licencia para EndNoteX6 (30 dólares) y la compra de un dominio para el blog Universo Abierto (30 dólares). En cuanto a la necesidad de personal, el servicio lo mantiene una única persona con dedicación completa de ocho horas diarias, quien además realiza otros servicios de la biblioteca.

Una particularidad de las tecnologías 2.0 es que se trata de herramientas que disponen de una gran potencialidad y que simplifican mucho la capacidad de uso, lo cual permite que un usuario medio pueda utilizarlas sin dificultad y que en muchos casos el éxito dependa sobre todo de la sagacidad del gestor para integrar diferentes herramientas en la creación de un servicio de información.

El origen de todos estos servicios data de finales de la década de 1980 con la aplicación del gestor de referencias ProCite (Alonso Arévalo, 2006), una de las primeras herramientas de gestión de referencias para recopilar información, fundamentalmente de artículos de revista, actas de congresos y libros, que buscaba disponer de un mecanismo único que permitiera apoyar las tareas de servicio de información y referencia de una manera eficiente. En aquellos momentos se disponía de un catálogo OPAC y más de 12 paquetes de revistas con distintas interfaces y mecanismos de búsqueda diferentes (ACS, Ebsco, Emerald, IEEE, IOP, JSTOR, KluwerLaw, Nature, Ovid, RSC, ScienceDirect, Springer, Swets y Wiley), lo que sin duda ralentizaba la búsqueda de información para los usuarios. La aplicación de ProCite consistía en recopilar la información de las distintas fuentes e integrarla en ProCite. Este gestor permitía recopilar fácilmente la información de referencia y exportarla en formato RIS. La información consistía fundamentalmente en artículos de revistas y se utilizaba para varios fines del servicio de información. El primero, y más básico, era dar respuesta eficiente a las cuestiones planteadas por cualquier usuario que necesitaba disponer de documentación actual y de calidad sobre un tema determinado. De este modo, cualquier persona que se dirigiera al servicio podría disponer, en unos pocos minutos, de un listado de las referencias, con descriptores, resumen del contenido y enlace hacia el documento en texto completo de todos los paquetes de revistas especializadas de que disponía la biblioteca. Este listado se enviaba por correo y el usuario sólo tenía que revisarlo.

Al incluir los enlaces a los artículos se facilitaba el acceso con un solo clic. Esta información servía también para crear un servicio de alerta informativa, 
que se remitía a los usuarios de manera periódica para mantenerlos informados sobre las novedades que de manera semanal se recibían en la biblioteca. A partir de esta base de datos se realizaban monográficos sobre cualquier tema, incluyendo todas las referencias que trataran sobre un asunto particular. En cuarto y último lugar servía para establecer un servicio de difusión selectiva de información para nuestros investigadores, a quienes cada trimestre se les enviaban las nuevas referencias de los artículos de su área de conocimiento. Por lo tanto se hacía una explotación de este recurso desde varias perspectivas (Figura 2): una explotación horizontal que es el Servicio de Alerta Informativa, otra explotación vertical que es la utilización para la elaboración de boletines monográficos sobre un tema, y otras dos transversales que responderían a las demandas al servicio de referencia y el servicio de DSI (Difusión Selectiva de Información) a instancias personales de usuarios determinados.

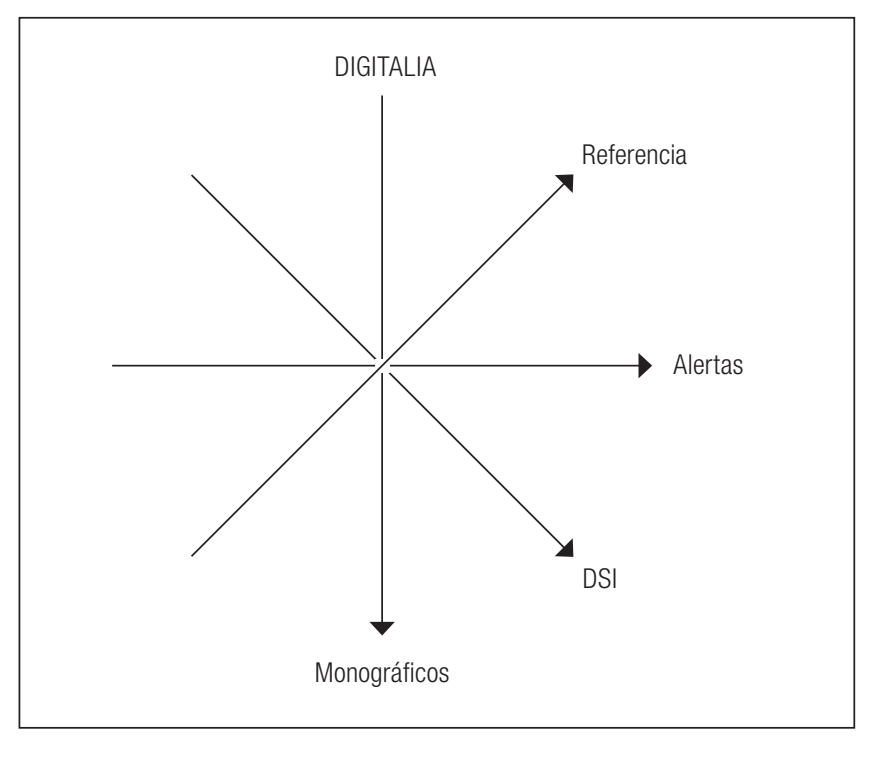

Figura 2. Recursos y su uso desde diferentes perspectivas.

Con el tiempo se vio la necesidad de difundir este trabajo para que, además de favorecer las tareas del servicio de referencia, permitiera adelantarnos a las necesidades de información de los usuarios ofreciéndoles un servicio de alertas. De esta manera nació la lista de distribución InfoDoC, primero distribuida con Lyris y actualmente a través de RedIris, la más importante red de información universitaria de España. Posteriormente se consideraron nuevos servicios más allá de los meramente bibliográficos y se amplió el uso 
de la lista de distribución para atender otras necesidades de información, como por ejemplo los cursos y congresos que se anunciaban o las ofertas de empleo para bibliotecarios y documentalistas. Esta diversificación en los servicios de información favoreció que la lista tuviera una amplia aceptación por parte de la comunidad de profesionales de la información en España, e incluso en buena parte de los países de habla hispana de América Central y de Latinoamérica, convirtiéndose así en la lista de distribución con más usuarios de la orbe hispana en Información y Documentación.

Con estos precedentes, la llegada de herramientas 2.0 en los primeros años de este siglo permitió seguir realizando aquello que se venía haciendo, pero optimizándolo con herramientas participativas y gratuitas (freeware u open source) que permitían mejorar la capacidad de llegar más y mejor a las personas interesadas. Una de las herramientas más utilizadas en el ámbito profesional son los blogs, por ello desde la biblioteca se consideró interesante la creación de uno.

El blog Universo Abierto se ha convertido en uno de los más populares en España en el ámbito de la Información y la Documentación; sus contenidos tratan sobre recursos, servicios y tecnologías de la información y ha tenido una progresión interesante: en 2009 se alcanzaron las 70000 visitas contadas por Google Analytics, en el año 2011 las 140000 y en 2012 fueron 270000 las visitas que permanecieron más de dos minutos en las páginas del blog. Un factor importante es la utilización de las redes sociales Facebook y Twitter para difundir los contenidos del blog, junto con la lista de distribución (Secker, 2008).

Otra herramienta 2.0 que presta una interesante utilidad es el escritorio Netvibes, un mashup que se utiliza para desarrollar una auténtica guía de recursos de la biblioteca. Quizás una de las mejores capacidades de la misma es la utilización de canales RSS, que permiten la integración de los diferentes contenidos. Estos están organizados en diferentes pestañas y la actualización de los mismos se va haciendo en tiempo real, con lo que en la pestaña "Revistas electrónicas", donde se integran las revistas gracias a los canales RSS de cada una de ellas, la información se va actualizando de manera automática cada vez que aparece un nuevo número o volumen de la revista. Lo mismo ocurre para otro tipo de información, como las nuevas adquisiciones de libros, los nuevos empleos, las noticias de prensa sobre un tema específico, etc. Se trata por tanto de una herramienta muy operativa, ya que se diseña una única vez y no es necesario invertir tiempo en la actualización de los contenidos. Incluso es posible añadir algunos widgets de Netvibes que permitan integrarla en la página principal de la biblioteca y funcionar como un gestor de contenidos, para que cualquier persona pueda ir viendo las entradas en el blog sin necesidad de ir a éste. 
Posteriormente se empezaron a utilizar gestores de referencias de segunda generación como fue el caso de Zotero, que además era un recurso de código abierto que permitía de una manera muy básica crear redes sociales en torno a aquellos temas de más actualidad. De este modo se empezaron a crear grupos en Zotero en torno a temas de interés, tales como Bibliotecas 2.0, Alfabetización informacional, Software libre, etc. Aunque tuvieron cierto nivel de funcionamiento estos grupos no lograron la repercusión deseada. Posteriormente se comenzó a trabajar con CiteUlike, una herramienta ya del entorno web 2.0 con mejores funcionalidades para crear grupos y poder compartir referencias con prestaciones de canales RSS y más capacidad para gestionar una comunidad de usuarios en torno a los temas que se habían planteado. En nuestra labor experimental se empezó a trabajar con Mendeley en 2009, que ofrecía aún mejores medios para crear una red de usuarios en torno a los temas que considerábamos más emergentes para el área de Información y Documentación. Estos grupos siguen existiendo en la red, con un seguimiento aceptable.

Desde este primer inicio de una red social especializada a partir de las referencias bibliográficas se consideró la posibilidad de trasladar nuestro proyecto al entorno de las redes sociales, donde ya se difundían algunos de los productos de la biblioteca, que se enviaban a la red desde Twitter y Facebook. El siguiente paso era recrear aquellos grupos que se habían abierto en la red social del gestor de referencias Mendeley en una red social de carácter generalizado y alta popularidad. Así se crearon diferentes grupos de Facebook, más dinámicos y participativos, en concreto los siguientes grupos profesionales de bibliotecas:

- Libros electrónicos (15 026 miembros) https://www.facebook.com/groups/universoebook/

- Software libre para bibliotecas (5 710 miembros) https://www.facebook.com/groups/softwarefree/

- ALFIN: Alfabetización Informacional (3 292 miembros) https://www.facebook.com/groups/347127501985354/

- Evaluación de la Investigación Científica (768 miembros) https://www.facebook.com/groups/351683134853230/

- Empleo en Biblioteconomía y Documentación (2 469 miembros) https://www.facebook.com/groups/Empleobyd/

- Traductores e intérpretes (14 298 miembros) https://www.facebook.com/groups/187330327976156/

- Somos 2.0 - Bibliotecas 2.0 (1 974 miembros) https://www.facebook.com/groups/somos20/ 
- Libros que recomendarías a un amigo mientras tomas un café (lectura social) (10 151 miembros)

https://www.facebook.com/groups/286482474746680/

- Deja una cita (1 096 miembros) https://www.facebook.com/groups/284230408311592/

- LLAAR (Lista Latinoamericana sobre Acceso abierto y Repositorios) (919 miembros)

http://www.facebook.com/groups/184675074889032

Como se puede apreciar por el número de seguidores los grupos tienen un importante dinamismo, lo cual ha suscitado el interés de buena parte de la comunidad profesional en torno a temas que consideramos que tienen gran relevancia en nuestra área de conocimiento. Los grupos son un recurso abierto a cualquier usuario y la experiencia muestra que son muy participativos. Es necesario tener en consideración que en cada grupo existe un número reducido de participantes altamente activos y una mayoría menos participativa, pero que sigue el desarrollo del grupo con interés, incluso después de casi dos años de funcionamiento sigue manteniéndose fiel al mismo. Para nuestra entidad supone un éxito ya solamente en cuanto a participación y seguidores, la suma total ronda casi los 7000 miembros entre todos los grupos, si bien hay usuarios que están en varios a la vez. Además consideramos que se trata de un mecanismo óptimo para difundir la información que se genera a través de otras herramientas, como es el caso de los posts del blog de la biblioteca, las listas de distribución, los monográficos y las novedades de adquisiciones en la biblioteca que difundimos a través del grupo de interés concreto, de manera que las cuestiones relativas a Software libre en bibliotecas las redifundimos a través de Facebook en este grupo; dichas intervenciones se replican automáticamente en Twitter. De esta manera se crea un entramado que retroalimenta unos recursos desde otros (Figura 3), de manera que se abren diferentes canales de comunicación tanto para promocionar nuestros recursos y servicios como para crear mecanismos de comunicación activos entre los investigadores de nuestra disciplina. 


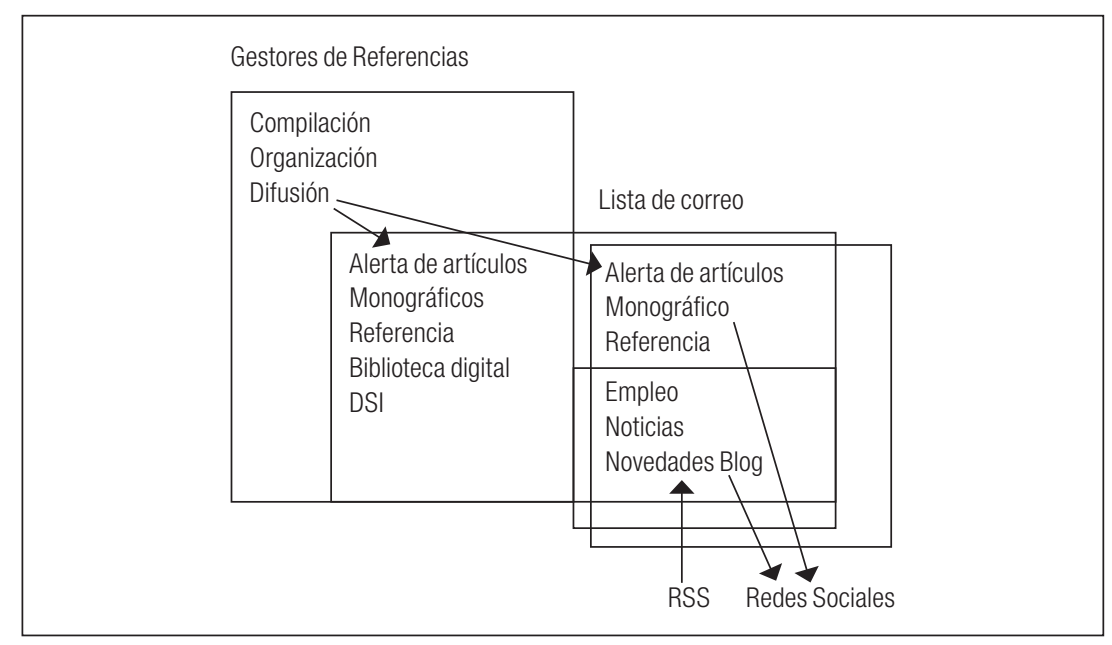

Figura 3. Integración de las herramientas 2.0 en un sistema de información

\section{Conclusiones}

1. Las bibliotecas constituyen poderosas instituciones para la canalización y la difusión de la información científica, principalmente cuando utilizan herramientas de la web 2.0 para promocionar sus servicios entre sus usuarios y para la difusión de contenidos, como los blogs y las redes de carácter general (Kim \& Abbas, 2010).

2. La integración de herramientas de diversa naturaleza entraña dificultades añadidas para el profesional, que ha de acometerlas con la convicción de que los mecanismos de creación de contenidos y la organización de la información, la difusión y la creación de canales de comunicación permiten una multiplicación de los beneficios para los usuarios, al mismo tiempo que favorecen que la biblioteca llegue a otros usuarios que no podrían acudir al centro, dotando a ésta de una visibilidad que redunda en beneficio de la institución y de la comunidad a la que atiende. La implementación de esta filosofía de trabajo permite la integración de los usuarios en los procesos de recopilación, mejora y validación de la información, favoreciendo mecanismos de participación en sintonía con las corrientes más novedosas de socialización del conocimiento.

3. La experiencia aportada por una biblioteca universitaria como la de la Facultad de Traducción y Documentación de la Universidad de Salamanca puede servir como referente extrapolable a otros contextos, 
en tanto que las herramientas y procesos desarrollados están disponibles para todos aquellos que pretendan crear un ecosistema informativo participativo y dinámico. Las bibliotecas y centros de información pueden encontrar en esta experiencia un referente probado de servicios bibliotecarios que integran diferentes herramientas con un mínimo coste material y humano y con grandes posibilidades de rentabilidad en términos de servicio y de marca de identidad.

4. La experiencia demuestra que el profesional de la información puede aportar importantes resultados a su entorno docente e investigador, integrarse en los procesos e inercias propias del área científica y pedagógica donde trabaja, y obtener reconocimiento profesional y social.

5. La virtualización de la mayoría de los servicios bibliotecario se entiende y justifica en un entorno en el que los usuarios han crecido y desarrollado sus capacidades cognitivas y sus destrezas en un contexto completamente tecnológico, en el que lo digital va desplazando a lo analógico (libros de texto, papel, etc.) como herramienta educativa. Entre las capacidades nuevas desarrolladas se encuentran: mayor inteligencia visual, gusto por la hipertextualidad o acceso no lineal a la información, inmediatez, mayor capacidad de resolución de problemas sin necesidad de consultar manuales y mayor capacidad de socialización en red.

6. Los servicios desarrollados por la biblioteca profesional -recordemos que es la biblioteca de los bibliotecarios de la Facultad de Traducción y Documentación- la han convertido en referencia para otros servicios bibliotecarios de la Universidad y de la propia Comunidad Autónoma de Castilla y León, que han seguido muchas de las pautas que se comenzaron a desarrollar en ella hace más de 20 años y que se han ido desarrollando a medida que se ofrecían nuevas herramientas y oportunidades de integración.

7. Desde la biblioteca se ha pretendido dar respuesta a esta nueva realidad con el desarrollo de un conjunto de herramientas y servicios que sitúan al usuario en el centro del ecosistema informativo. La biblioteca funciona como lugar de encuentro para la creación, producción y difusión de la información, pero también como lugar de estímulo para la participación y el conocimiento compartido. A través de esta experiencia en marcha se ha comprobado la necesidad de que los profesionales integren nuevas competencias en su desempeño profesional y de que la biblioteca se erija en una suerte de panóptico invertido en el que los usuarios observen y centrifuguen sus intereses para que sean replicados, mejorados o conservados por todos aquellos que los comparten. 
8. Una de las tareas inherentes a cualquier servicio de información es la transmisión de los conocimientos acumulados. La experiencia de estos años se ha recogido y transmitido en forma de Alfabetización Informacional; es evidente que la concreción de un servicio de calidad entre nuestros usuarios favorece claramente su confianza en recurrir a la biblioteca como mecanismo de formación de alumnos, profesores y personal de administración. Además, al formarse en la facultad los futuros profesionales bibliotecarios, la biblioteca vela por alcanzar la calidad y buenas prácticas profesionales que puedan servir de modelo para los futuros egresados. Los planes de formación articulados desde la biblioteca para dotar a los nuevos alumnos de conocimientos y autonomía en la resolución de sus necesidades informativas responden a esta filosofía. La biblioteca se integra en todos los niveles docentes de las diferentes titulaciones que se imparten, desde el Grado a los Masters, con objeto de facilitar una formación completa y de calidad en materia informativa, participando igualmente con aquellos equipos y grupos de investigación que trabajan en ella.

9. Todo profesional debe poseer las competencias necesarias para utilizar e interactuar con herramientas que permitan generar un sistema que sirva para compilar, organizar y difundir la información, para hacerla más accesible a las necesidades e intereses de los usuarios, tanto directos como indirectos, ya que estos nuevos medios sirven para poder llegar a más personas con el mismo esfuerzo que tendríamos que hacer para llegar a nuestros usuarios presenciales. La red abre estas posibilidades y nos allega la oportunidad para aprovecharlas y demostrar que el trabajo profesional, lejos de parecer innecesario, se renueva constantemente y se encuentra presente en el día a día de nuestros usuarios.

\section{Bibliografía}

Aguillo, I. (2009), "Measuring the institution's footprint in the web", en Library Hi Tech, vol. 27, núm. 4, pp. 540-556. Disponible en: http://www.emeraldinsight.com/10.1108/073788309

Aharony, N. (2009), "Uso de la web 2.0 por los bibliotecarios", en Boletín de la Asociación Andaluza de Bibliotecarios, vol. 24, núm. 9697, pp. 129-160. Disponible en: http://www.aab.es/pdfs/baab969 7/96-97col07.pdf 
Alonso Arévalo, J. (2006), "ProCite® 5.0: Guía”, en Biblioteca Virtual en Ciencias de la Salud. Habilidades informacionales: recursos, metodología y técnicas de trabajo científico. Disponible en: http://eprints. rclis.org/archive/00007214/01/ProCite50.pdf

__; Cordón García, J. A. \& Martín Rodero, H. (2010), “CiteULike y Connotea: herramientas 2.0 para el descubrimiento de la información científica", en El Profesional de la Información, vol. 19, núm. 1, pp. 86-94. Disponible en: http://elprofesionaldelainformacion.me tapress.com/openurl.asp?genre=article $\&$ id $=$ doi:10.3145/epi.2010. ene.12

— \& Vázquez Vázquez, M. (2013), "Las listas de distribución como servicio de información: InfoDoc”, en Mi Biblioteca, vol. 9, núm. 34, pp. 58-67.

Armstrong, A. (2011), "Searching 2.0", en The Journal of Academic Librarianship, vol. 37, núm. 2, p. 187. Disponible en: http://www.sciencedirect.com/science/article/pii/S0099133311000139

Baiget, T. (2010), "Profesionales de la información: un futuro de oportunidades”, en E-LIS: E-Prints in Library and Information Science. Disponible en: http://eprints.rclis.org/19323/

Brody, T. (2006), "Evaluating Research Impact through Open Access to Scholarly Communication", en ECS EPrints Repository, Disponible en: http://eprints.ecs.soton.ac.uk/13313/1/brody.pdf

Burgelman, J.-C.; Osimo, D. \& Bogdanowicz, M. (2010), “Science 2.0 (change will happen...)", en First Monday, vol. 15, núm. 7. Disponible en: http://www.uic.edu/htbin/cgiwrap/bin/ojs/index.php /fm/article/viewArticle/2961/2573

Casey, M. E. \& Savastinuk, L. C. (2006), “Library 2.0”, en Library Journal, vol. 131, núm. 14, pp. 40-42. Disponible en: http://lj.library journal.com/2010/05/technology/library-2-0/

Castagné, M. (2011), Rural Library 2.0: A Proposal to Measure the Success of Web 2.0 Technologies in Michigan's Rural Public Libraries, San José: San José State University. Disponible en: http://www. vcn.bc.ca/ castagne/papers/rural_library.pdf

Cooke, N. A. (2011), "Professional development 2.0 for librarians: Developing an online personal learning network (PLN)", en Proceedings of the 77th IFLA General Conference and Assembly (San Juan, Puerto Rico, 2011), The Hague, Netherlands: IFLA, pp. 1-13. Disponible en: http://conference.ifla.org/past/ifla77/200-cooke-en.pdf

Cordón-García, J. A.; Alonso-Arévalo, J.; Gómez-Díaz, R. \& López Lucas, J. (2012a), Las nuevas fuentes de información: información y búsqueda documental en el contexto de la web 2.0, Madrid: Pirámide.

- ; Carbajo Cascón, F.; Gómez Díaz, R. \& Alonso Arévalo, J. (2012b), Libros electrónicos y contenidos digitales en la sociedad del conocimiento: Mercado, servicios y derechos, Madrid: Pirámide, ISBN 978-84-3682-769-9. 
Cordón García, J. A.; Gómez Díaz, R. \& Alonso Arévalo, J. (2012c), Gutenberg 2.0: la revolución de los libros electrónicos, Gijón: Trea, ISBN 978-84-9704-634-3.

—; Alonso-Arévalo, J.; Gómez-Díaz, R. \& Alonso Berrocal, J. L. (2014), El Ecosistema del Libro Electrónico Universitario, 2a ed. rev. y act., Madrid: UNE, ISBN 978-84-9012-286-0.

Chad, K. \& Miller, P. (2005), Do libraries matter? The rise of Library 2.0, London: Talis. Disponible en: http://www.capita-libraries. co.uk/downloads/white_papers/DoLibrariesMatter.pdf

Chapman, A. \& Russell, R. (2009), "Collecting Evidence in a Web 2.0 Context”, en Ariadne, núm. 60. Disponible en: http://www.ariad ne.ac.uk/issue60/chapman-russell/

Daniels, J. \& Roth, P. (2012), "Incorporating Millennium Catalog Records into Serials Solutions' Summon”, en Technical Services Quarterly, vol. 29, núm. 3, pp. 193-199. Disponible en: http://dx.doi. org/10.1080/07317131.2012.681283

Feng, A. (2011), Corporate Librarian 2.0: New Core Competencies, Alexandria, Virginia: Special Libraries Association (SLA). Disponible en: http://units.sla.org/division/dpht/division_info/travel-presentations/feng_essay.pdf

González-Fernández Villavicencio, N. (2007), "Bibliotecas 2.0 en España (el camino recorrido)", en Boletín de la Asociación Andaluza de Bibliotecarios, vol. 22, núm. 86-87, pp. 29-46. Disponible en: http://www.aab.es/pdfs/baab86-87/86-87a2.pdf

Harris, L. E. (2008), "Social software in libraries: Building collaborations, communication, and community online", en Journal of the American Society for Information Science and Technology, vol. 59, núm. 9. Disponible en: http://dx.doi.org/10.1002\%2Fasi.20867

Holmberg, K.; Huvila, I.; Kronqvist-Berg, M. \& Widén-Wulff, G. (2009), "What is Library 2.0?", en Journal of Documentation, vol. 65, núm. 4, pp. 668-681. Disponible en: www.emeraldinsight. com/10.1108/00220410910970294

Jacsó, P. (2011), "Traditional scholarly publishers and web 2.0”, en Online Information Review, vol. 35, núm. 2, pp. 301-315. Disponible en: http://www.emeraldinsight.com/journals.htm?articleid=1917498

Jiepu Jiang, D. H. \& Ni, C. (2011), "Social Reference: Aggregating Online Usage of Scientific Literature in CiteULike for Clustering Academic Resources”, en JCDL'11 (Ottawa, Ontario, Canadá, 1317 junio, 2011), vol. 11, pp. 401-402. Disponible en: http://www. sis.pitt.edu/ jjiang/papers/jcdl.social.reference.pdf

Kim, Y.-M. \& Abbas, J. (2010), "Adoption of Library 2.0 Functionalities by Academic Libraries and Users: A Knowledge Management Perspective", en The Journal of Academic Librarianship, vol. 36, núm. 3, pp. 211-218. Disponible en: http://www.sciencedirect. com/science/article/B6W50-4YRXKGN-1/2/8009ac31749faac0 8046afa10d276c4c 
Kroski, E. (2008), Web 2.0 for Librarians and Information Professionals, New York: Neal-Schuman, ISBN 978-1-55570-614-2.

Méndez González, R. (2012), Traducción E paratraducción de videojuegos: textualidad y paratextualidad en la traducción audiovisual y multimedia, Vigo: Universidad de Vigo, Facultad de Traducción e Interpretación. Disponible en: http://www.paratraduccion.com/ docu/Tesis_Ramon/Portada-Intro_Tesis-Doctoral_Ramon.pdf

Miller, P. (2005), "Web 2.0: Building the New Library", en Ariadne, núm. 45. Disponible en: http://www.ariadne.ac.uk/issue45/miller/

Neylon, C. (2011), "Re-use as Impact: How re-assessing what we mean by 'impact' can support improving the return on public investment, develop open research practice, and widen engagement", en altmetrics11: Tracking scholarly impact on the social Web (Koblenz, Germany, 14-15 junio, 2011), ACM Web Science Conference 2011 Workshop. Disponible en: http://altmetrics.org/ workshop2011/neylon-v0/

Nguyen, L. C.; Partridge, H. \& Edwards, S. L. (2012), "Towards an understanding of the participatory library", en Library Hi Tech, vol. 30, núm. 2, pp. 335-346. Disponible en: http://eprints.qut. edu.au/50107/1/Nguyen_Partrige_Edwards_Library_Hi_Tech_ Author_Copy_2012.pdf

Noa, A. (2012), "An Analysis of American Academic Libraries Websites: 2000-2010", en The Electronic Library, vol. 30, núm. 6, pp. 764-776. Disponible en: http://links.emeraldinsight.com/journals.htm?articleid $=17053619$

Priem, J. \& Hemminger, B. H. (2010), "Scientometrics 2.0: Toward new metrics of scholarly impact on the social Web", en First Monday, vol. 15, núm. 7. Disponible en: http://firstmonday.org/htbin/ cgiwrap/bin/ojs/index.php/fm/article/view/2874/2570

_; Piwowar, H. A. \& Hemminger, B. H. (2011), "Altmetrics in the wild: An exploratory study of impact metrics based on social media", en Metrics 2011: Symposium on Informetric and Scientometric Research (New Orleans, LA, USA, 2011). Disponible en: http://jasonpriem.org/self-archived/PLoS-altmetrics-sigmetrics11-abstract.pdf

Quinney, K.; Smith, S. \& Galbraith, Q. (2010), "Bridging the Gap: Self-Directed Staff Technology Training", en Information Technology and Libraries, vol. 29, núm. 4, pp. 205-213. Disponible en: http://www.ala.org/lita/ital/files/29/4/quinney.pdf

Rasmussen, L. (2009), "The Librarian 2.0 and the visual Information Professional", en Proceedings of the BOBCATSSS 2009 (Porto, Portugal, 28-30 enero, 2009), Ankara, Turkey: EUCLID (European Association for Library \& Information Education and Research). Disponible en: http://eprints.rclis.org/bitstream/10760/12936/1/122.pdf

REBIUN, R.D.B.U.E. (2011), Science 2.0: The Use of Social Networking in Research, REBIUN. Disponible en: http://eprints.rclis.org/bitstream/10760/16162/1/Science20_rebiun_2011.pdf 
Rozaklis, L. \& Macdonald, C. M. (2011), "A Typology of Collaborative Communication in a Digital Reference Environment", en The Reference Librarian, vol. 52, núm. 4, pp. 308-319. Disponible en: http://dx.doi.org/10.1080/02763877.2011.586907

Saha, N. C.; De, S. \& Paul, N. (2008), "Application Of Web 2.0 In Library And Information Science: With Special Reference To RSS", en Proceedings of the 6th Convention PLANNER (Nagaland, 2008), Nagaland University. Disponible en: http://ir.inflibnet.ac.in/dx$\mathrm{ml} /$ bitstream/handle/1944/1157/37.pdf? sequence $=1$

Secker, J. (2008), "Social software and libraries: a literature review from the LASSIE project", en Program: electronic library and information systems, vol. 43, núm. 2, pp. 215-231. Disponible en: http://eprints. lse.ac.uk/20338/2/Social_software_and_libraries_(LSERO)_.pdf

Spring, H. (2011), "If you cannot beat them, join them! Using Health 2.0 and popular Internet applications to improve information literacy", en Health Information \& Libraries Journal, vol. 28, núm. 2, pp. 148-151. Disponible en: http://dx.doi.org/10.1111/j.1471-1842. 2011.00934.x

Stephens, M. (2006), "Web 2.0 and Libraries: Best Practices for Social Software", en Library Technology Reports, vol. 42, núm. 4.

Tajer, P. (2009), "Reference Services 2.0: A Proposal Model for Reference Services in Library 2.0", en Proceedings of the 7th International CALIBER-2009 (Pondicherry University, Puducherry, febrero 2527, 2009). Disponible en: http://www.inflibnet.ac.in/caliber2009/ CaliberPDF/38.pdf

Thelwall, M. (2008), "Social networks, gender, and friending: An analysis of MySpace member profiles", en Journal of the American Society for Information Science and Technology, vol. 59, núm. 8, pp. 1321-1330. Disponible en: http://ejournals.ebsco.com/direct.asp? ArticleID $=4$ A8A98A753F09E6497F1

Tripathi, M. \& Kumar, S. (2010), "Use of Web 2.0 tools in academic libraries: A reconnaissance of the international landscape", en The International Information \& Library Review, vol. 20, pp. 195-207. Disponible en: http://ubiblioteca.unbosque.edu.co/Plan\%20de\% 20desarrollo/Use\%20of\%20Web\%202.0\%20tools\%20in\%20aca demic\%20libraries.pdf

Vállez, M. \& Marcos, M.-C. (2009), "Las bibliotecas en un entorno Web 2.0", en Hipertext.net, núm. 7. Disponible en: http://www.hipertext.net/web/pag298.htm

Wilson, T. (2012), "The Kept-Up Academic Librarian: Helping Academic Librarians 'Keep Up' With News and Developments In Higher Education -http://keptup.typepad.com", en Technical Services Quarterly, vol. 29, núm. 3, pp. 246-247. Disponible en: http://dx.doi.org/10.1080/07317131.2012.682019 